\title{
Interrelationship between Changes in the Amyloid $\beta$ 42/40 Ratio and Presenilin 1 Conformation
}

\author{
Katarzyna Marta Zoltowska, Masato Maesako, and Oksana Berezovska
}

MassGeneral Institute for Neurodegenerative Disease, Massachusetts General Hospital, Harvard Medical School, Department of Neurology, Charlestown, Massachusetts, United States of America

\begin{abstract}
The ratio of the longer $(A \beta 42 / A \beta 43)$ to shorter $(A \beta 40)$ species is a critical factor determining amyloid fibril formation, neurotoxicity and progression of the amyloid pathology in Alzheimer's disease. The relative levels of the different A $\beta$ species are affected by activity and conformation of the $\gamma$-secretase complex catalytic component presenilin 1 (PS1). The enzyme exists in a dynamic equilibrium of the conformational states, with so-called "close" conformation associated with the shift of the $\gamma$-secretase cleavage toward the production of longer, neurotoxic A $\beta$ species. In the current study, fluorescence lifetime imaging microscopy, spectral Förster resonance energy transfer, calcium imaging and cytotoxicity assays were utilized to explore a reciprocal link between the $A \beta 42$ and $A \beta 40$ peptides present at various ratios and PS1 conformation in primary neurons. We report that exposure to $A \beta$ peptides at a relatively high ratio of $A \beta 42 / 40$ causes conformational change within the PS1 subdomain architecture toward the pathogenic "closed" state. Mechanistically, the A $342 / 40$ peptides present at the relatively high ratio increase intracellular calcium levels, which were shown to trigger pathogenic PS1 conformation. This indicates that there is a reciprocal cross-talk between the extracellular A $\beta$ peptides and PS1 conformation within a neuron, with $A \beta 40$ showing some protective effect. The pathogenic shift within the PS1 domain architecture may further shift the production of A $\beta$ peptides toward the longer, neurotoxic $A \beta$ species. These findings link elevated calcium, A 342 and PS1/ $\gamma$-secretase conformation, and offer possible mechanistic explanation of the impending exacerbation of the amyloid pathology.
\end{abstract}

Online address: http://www.molmed.org

doi: $10.2119 /$ molmed.2016.00127

\section{INTRODUCTION}

Alzheimer's disease (AD) is a neurodegenerative disorder characterized by accumulation of the extracellular amyloid $\beta(\mathrm{A} \beta)$ aggregates in the brain, as one of the major pathological hallmarks of the disease $(1,2)$. The presenilin 1 (PS1) $/ \gamma$-secretase cleavage releasing A $\beta$ can occur at several positions within the transmembrane region of APP, resulting in production of a mixture of peptides varying in their length and aggregation properties (3-5). The majority of the peptides produced constitute the $A \beta 40$ species. However, greater emphasis has been placed on the longer $A \beta 42 / 43$ peptides that are detected at approximately 10 -fold lower levels than A $\beta 40$. The longer $\mathrm{A} \beta$ peptides have strong neurotoxicity and intrinsic self-assembly properties, promoting their accumulation in the extracellular amyloid plaques $(6,7)$.

Importantly, the vast majority of mutations in APP and PS1 shift the

Address correspondence to Oksana Berezovska. MassGeneral Institute for

Neurodegenerative Disease, Massachusetts General Hospital, Harvard Medical School, Department of Neurology, $11416^{\text {th }}$ Street, Charlestown, MA 02129. Phone: 1-617-726-7478; Fax: 1-617-724-1480; E-mail: oberezovska@partners.org

Submitted May 10, 2016; Accepted for publication July 5, 2016; Published Online (wWw.molmed.org) July 5, 2016.

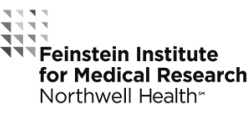

spectrum of $A \beta$ peptides toward the longer species (http:/ / www.alzforum. org/mutations) (8). A small elevation in the $A \beta 42 / 40$ ratio increases $A \beta$ peptide's aggregation properties, induces neurotoxicity and alters synaptic activity in primary neurons (9). On the other hand, it was suggested that $A \beta 40$ might serve as a protective factor in AD progression, mediating the inhibition of $A \beta 42$ fibrillogenesis (10). Collectively, these data suggest that elevated $A \beta 42 / 40$ ratio is linked to AD pathogenesis and could be targeted therapeutically.

The elevation of the $A \beta 42 / 40$ ratio in the pathogenic condition might be attributed to the conformational shift in PS1, a catalytic subunit of the $\gamma$-secretase complex. The mature form of the enzyme can adopt different conformational states, as determined by the single particle electron microscopy and fluorescence lifetime imaging (FLIM) $(11,12)$. The close PS1 domain proximity, as it is observed in the familial AD 
mutant PS1, favors $\gamma$-secretase cleavage site(s) on APP, producing longer A $\beta$ species (13). Hence, we refer to this state as "closed," pathogenic PS1 conformation. Importantly, pathogenic PS1 conformation, elevated production of the longer, fibrillogenic $A \beta$ species and consequent increase in the $A \beta 42 / 40$ ratio, leading to gradual amyloid deposition, occur during aging, and in sporadic AD brains (14-16). Of note, the occurence of PS1 conformational change correlates with the proximity to $A \beta$ plaques (15). On the other hand, compounds that allosterically modulate PS1 by shifting it toward the "open" conformation consequently shift the spectra of $A \beta$ species toward the shorter peptides $(11,17,18)$. This suggests that PS1 conformational change is upstream of the various amyloid $\beta$ specie production. However, it remains unclear whether there is a reciprocal cross-talk between A $\beta$ and PS1/ $\gamma$-secretase conformational changes.

This study investigates a mechanistic link between the high $\mathrm{A} \beta 42 / 40$ ratio and PS1 conformation. We found that a mixture of $A \beta 40$ and $A \beta 42$ peptides at the relatively high $A \beta 42 / 40$ ratio can reciprocally modulate PS1 conformation by shifting it toward the pathogenic state. We propose that this is mediated by increased intracellular calcium levels. Such conformational shift, in turn, results in a greater production of the $\mathrm{A} \beta 42$ relative to $A \beta 40$, and may promote further rapid cell-autonomous and noncellautonomous spread of the amyloid pathology within the brain.

\section{MATERIALS AND METHODS}

\section{Plasmids}

Adeno-associated viral (AAV) vector plasmid encoding presenilin 1 (PS1) with the green fluorescent protein (GFP) fused to the N-terminus and the red fluorescent protein (RFP) inserted in the loop domain, under hSyn1 promoter, was used as a reporter of the PS1 conformation. The plasmid was packaged in AAV serotype 8 capsid at University of Pennsylvania Vector Core.

\section{Primary Neuronal Cultures}

Mixed cortical primary neuronal cultures were obtained from cerebral cortex of CD1 wild type mouse embryos at gestation d 16-18. The dissected tissue was dissociated using Papain Dissociation Kit (Worthington Biochemical Corporation), the neurons were plated on poly-D-lysine coated dishes and cultured in Neurobasal Medium supplemented with $10 \%$ fetal bovine serum, 2\% B27, 1\% penicillin/streptomycin and 1\% Gluta$\max$ (ThermoScientific) in $37^{\circ} \mathrm{C}, 5 \% \mathrm{CO}_{2}$ incubator. When necessary, the neurons were infected at $5 \mathrm{~d}$ in vitro (DIV) with AAV viruses by addition of the respective AAV stocks into culture media, and $6 \mathrm{~d}$ incubation prior to the analysis.

All experiments involving animals were performed under national (United States National Institutes of Health) and institutional (Massachusetts General Hospital Subcommittee for Research Animal Care) approved guidelines.

\section{Amyloid $\beta$ Preparation and Treatment}

The A $\beta$ peptides were prepared as described previously $(9,19)$. Briefly, A $\beta 42$ and $A \beta 40$ peptides (Peptide Institute) dissolved at $1 \mathrm{mg} / \mathrm{mL}$ concentration in hexafluoroisopropanol (HFIP), were mixed in molar ratio of 1:9, 3:7, 4:6, 1:0, 4:0 and 10:0, respectively. HFIP was evaporated and the peptides were resuspended in PBS/2\% DMSO to the final concentration of $22 \mu \mathrm{mol} / \mathrm{L}$. Then the samples were filtered using $0.2 \mu \mathrm{m}$ Whatman Polyethersulfone (PES) Filter Puradiscs (GE Healthcare Bio-Sciences). The samples were kept on ice with the lag time maximum of $15 \mathrm{~min}$. For the treatment of primary neurons, the peptides were diluted in Neurobasal Medium to $1 \mu \mathrm{mol} / \mathrm{L}$ or $10 \mathrm{nmol} / \mathrm{L}$ final concentration and the incubation was continued for $2 \mathrm{~h}$ at $37^{\circ} \mathrm{C}$.

\section{Immunocytochemistry}

Neurons were fixed with $4 \%$ paraformaldehyde in phosphate-buffered saline (PBS), permeabilized with $0.1 \%$ Triton- $X$, and the nonspecific binding of antibodies was blocked by $1 \mathrm{~h}$ incubation with 1.5\% normal donkey serum (NDS) (Jackson ImmunoResearch Labs). Anti-PS1 N-terminus (Abcam) and anti-PS1 loop domain (Abcam) primary antibodies were applied overnight at $4^{\circ} \mathrm{C}$. Corresponding Alexa Fluor 488- and Cy3-conjugated secondary antibodies were used for detection. The slides were mounted using Vectashield mounting medium (Vector Laboratories Inc.).

\section{Fluorescence Lifetime Imaging Microscopy (FLIM)}

The relative proximity between fluorescently labeled N-terminus and the cytoplasmic loop domain between the transmembrane helices 6 and 7 of the endogenous PS1 was determined by previously validated FLIM assay for PS1 conformation (11). Briefly, pulsing Chameleon Ti:Sapphire laser (Coherent Inc.) was used to excite Alexa Fluor 488 donor fluorophore (two-photon excitation at 780-nm wavelength). The lifetimes of the donor fluorophore were recorded using a high-speed photomultiplier tube (MCP R3809; Hamamatsu) and a fast time-correlated single-photon counting acquisition board (SPC-830; Becker \& Hickl). The baseline lifetime of the donor fluorophore (Alexa Fluor 488) in the absence of the acceptor (Cy3) was determined as the Förster resonance energy transfer (FRET) negative control $(\tau 1)$. The presence of acceptor fluorophore $<10 \mathrm{~nm}$ from the donor results in FRET and a characteristic shortening of the donor fluorophore lifetime $(\tau 2)$. The degree of the donor lifetime shortening correlates with the relative proximity between the fluorophores. The percent FRET efficiency $\left(\mathrm{E}_{\mathrm{FRET}} \%\right)$ is calculated as follow: $\mathrm{E}_{\mathrm{FRET}} \%=(\tau 1-\tau 2) / \tau 1^{*} 100 \%$. The images were acquired using Zeiss LSM510 confocal microscope with ZEN 2009 software equipped with Zeiss 63x/1.4 Oil DIC objective. The data were analyzed using SPC Image software (Becker \& Hickl).

\section{Spectral FRET}

Spectral FRET assay was performed as described previously (20). Briefly, the GFP was excited using the Argon laser 
at $488 \mathrm{~nm}$ and emitted fluorescence was detected at $513 \pm 10.57 \mathrm{~nm}$ (GFP) and $598 \pm 10.57 \mathrm{~nm}$ (RFP) spectral bandwidth of the Metadector in the Zeiss LSM510 confocal microscope equipped with Zeiss 25x/0.8 Corr DIC objective, with ZEN 2009 software. Fluorescence intensity of individual neurons was measured using Image J $1.46 \mathrm{c}$ software. The ratio of the $598 \mathrm{~nm}$ to $513 \mathrm{~nm}$ fluorescence intensity was used as a readout of the FRET efficiency, that is, the relative proximity between the GFP- and RFP-labeling respective PS1 domains.

\section{Calcium Imaging}

Calcium influx was induced by the application of $50 \mathrm{mmol} / \mathrm{L} \mathrm{KCl}$ or $5 \mu \mathrm{mol} / \mathrm{L}$ calcium ionophore A2317 (Sigma Aldrich). The intracellular calcium levels were determined using ratiometric dye Indo-1, as described previously (21). Briefly, Indo-1/AM (ThermoScientific) was dissolved with $20 \%$ pluronic F-127 (ThermoScientific) in DMSO and added to the culture dishes at a final concentration of $1 \mu \mathrm{mol} / \mathrm{L}$ Indo- $1 / \mathrm{AM}$ and $0.02 \%$ pluronic F-27 for $45 \mathrm{~min}$. Cells were imaged with ZEN 2009 software using Zeiss LSM510 inverted confocal microscope equipped with Zeiss 25x/0.8 Corr DIC objective. The Chameleon Ti:Sapphire laser was used at 750-nm two-photon excitation, and the emitted light was discriminated into two channels with interference filters corresponding to 390 $\mathrm{nm}, 65-\mathrm{nm}$ bandpass and $495 \mathrm{~nm}, 20-\mathrm{nm}$ bandpass (Chroma Technology). The data was analyzed using ImageJ $1.46 \mathrm{c}$ software.

Alternatively, changes in the intracellular calcium level were determined using Oregon Green BAPTA-1/AM (ThermoScientific). The neurons were loaded with $5 \mu \mathrm{mol} / \mathrm{L}$ Oregon Green BAPTA-1/AM by 30-min incubation in $\mathrm{Ca}^{2+} / \mathrm{Mg}^{2+}$ Hank's Balanced Salt Solution (ThermoScientific). The dye was washed off and the cells were imaged with ZEN 2009 software using Zeiss LSM510 inverted confocal microscope equipped with Zeiss 25x/0.8 Corr DIC objective.

\section{Enzyme-Linked Immunosorbent Assay (ELISA)}

The abundance of the $A \beta$ oligomers that have at least two intact $\mathrm{N}$-termini in the $A \beta$ preparations was determined using amyloid- $\beta$ oligomers 82 E1-specific ELISA (IBL America) according the manufacturer's protocol.

\section{Dot Blot}

$\mathrm{A} \beta$ oligomers were detected using the dot blot method. Briefly, the A $\beta$ preparations were applied on the nitrocellulose membrane (GE Healthcare Lifesciences). The nonspecific binding was blocked using Odyssey Blocking Buffer (LI-COR) and the membranes were probed with the anti-amyloid oligomers A11 antibody (Abcam) and a corresponding secondary antibody (LI-COR). The signal was developed using the digital imaging system LI-COR Odyssey scanner.

\section{Cytotoxicity Assay}

The cytotoxicity was determined using a cytotoxicity detection kit (Sigma-Aldrich) according to the manufacturer's recommendation. Briefly, $50 \mu \mathrm{L}$ of the conditioned medium were mixed with $50 \mu \mathrm{L}$ of the reaction mixture and incubated in dark for $20 \mathrm{~min}$ at room temperature. The absorbance at $490 \mathrm{~nm}$ was read using a Wallac plate reader (PerkinElmer).

\section{Statistical Analysis}

Statistical significance was calculated with GraphPad Prism 5 software using a two-tailed unpaired Student $t$ test or Pearson's X-square test. $p$ value of $<0.05$ was a predetermined threshold for statistical significance.

All supplementary materials are available online at www.molmed.org.

\section{RESULTS}

\section{A $342 / 40$ Ratio Modulates PS 1 Conformation}

Fluorescence lifetime imaging microscopy (FLIM), combined with the immunohistochemical analysis of human sporadic AD (sAD) brains revealed that
PS1 exists in the "closed" pathogenic state in neurons proximal to the amyloid deposits (15). To determine whether an increase in the $A \beta 42$ and/or the A $\beta 42 / 40$ ratio can in turn modulate PS1 subdomain arrangement, we incubated primary neurons with $A \beta$ mixtures containing $A \beta 42$ to $A \beta 40$ in the following ratios: $1: 9 ; 3: 7 ; 4: 6 ; 10: 0$. The cells were treated for $2 \mathrm{~h}$ with the total $\mathrm{A} \beta$ at 1 $\mu \mathrm{mol} / \mathrm{L}$ final concentration. The applied A $\beta$ peptide preparations were characterized by oligomeric $A \beta$ enzyme-linked immunosorbent assay (ELISA) and dot blot with oligomeric $A \beta$ specific antibody (Figure 1). As expected, the increase in the $A \beta 42$ amount relative to $\mathrm{A} \beta 40$ resulted in the greater abundance of the oligomeric $A \beta$ species. Interestingly, the relatively small change in the A $\beta 42 / 40$ ratio from 3:7 to 4:6 resulted in a profound increase in the fibrillogenic properties of the $\mathrm{A} \beta$ peptides.

Following the treatment, endogenous PS1 conformation was analyzed by measuring relative proximity between the fluorophores labeling the PS1 Nterminus and the PS1 loop domain using antibody-based FLIM. There was no significant difference in the PS1 conformation between vehicle-treated neurons and those treated with the $A \beta 42 / 40$ at the $1: 9$ and 3:7 ratios. However, characteristic shortening of the donor fluorophore lifetime, indicative of the increased FRET efficiency, was detected when the cells were treated with the $A \beta 42 / 40$ at 4:6 and 10:0 ratios $(121 \pm 4 \%$ and $132 \pm$ $4 \%$, respectively, as compared with the 1:9 ratio) (Figure 2A). These data were further confirmed using a complementary approach: adeno-associated viral (AAV)-mediated overexpression of PS1 was tagged with green fluorescent protein (GFP) at the N-erminus and the red fluorescent protein (RFP) was inserted in the loop domain, and the determination of the proximity between the fluorophores was made using spectral FRET (Supplementary Figure S1). Similarly, higher FRET efficiency was recorded in the neurons pretreated with the $A \beta 42 / 40$ peptides at the 4:6 and 10:0 ratios. 
A

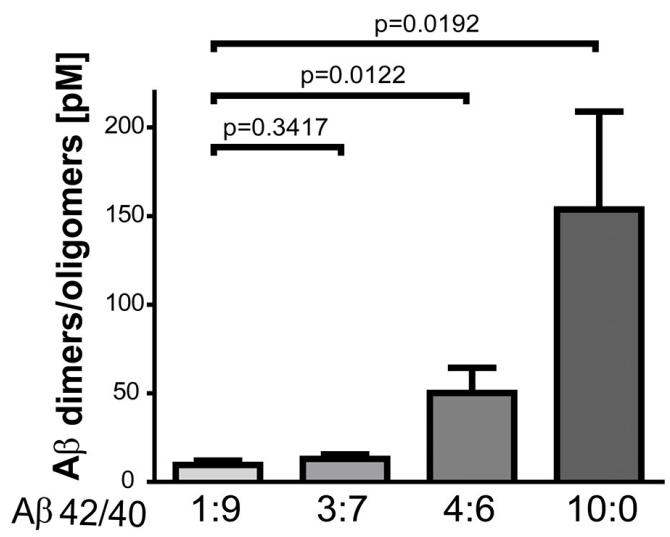

B

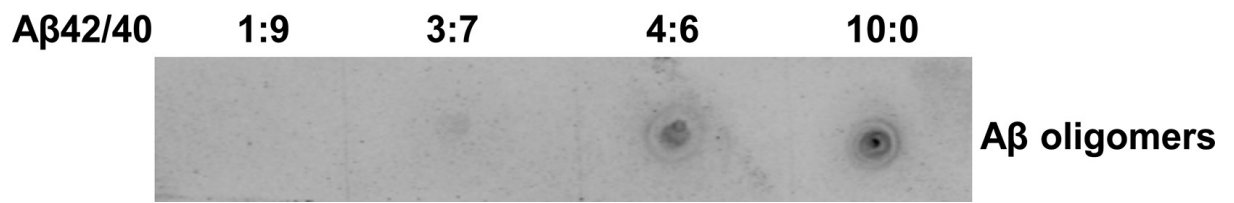

Figure 1. Characterization of $A \beta$ peptides. (A) ELISA analysis of the $A \beta$ dimers/oligomers in the mixtures of the $A \beta 42$ and $A \beta 40$ peptides mixed at the ratios of $1: 9,3: 7,4: 6$ and $10: 0$ at the total $1 \mu \mathrm{mol} / \mathrm{L} A \beta$ concentration. $n=9$, mean $\pm S E M$, two-tailed unpaired Student $t$ test. (B) The representative dot blot presents the abundance of $A \beta$ oligomers detected by $A \beta$ oligomer specific antibody in the mixtures of the $A \beta 42$ and $A \beta 40$ peptides mixed at the ratios of $1: 9,3: 7,4: 6$ and $10: 0$ at the total $1 \mu \mathrm{mol} / \mathrm{L} A \beta$ concentration. $A \beta$ - amyloid $\beta$.

These data suggest at least two possibilities: the conformational shift of PS1 toward the closed, pathogenic state may be induced either by higher $A \beta 42 / 40$ ratio or by higher $\mathrm{A} \beta 42$ concentration. To distinguish between these two possibilities, the neurons were treated with $A \beta 42$ and $A \beta 40$ peptides at the following ratios and relative amounts, respectively: 1:9 $(0.1 \mu \mathrm{mol} / \mathrm{L} \mathrm{A} \beta 42$ : $0.9 \mu \mathrm{mol} / \mathrm{L} \mathrm{A} \beta 40), 4: 6(0.4 \mu \mathrm{mol} / \mathrm{L} \mathrm{A} \beta 42$ : $0.6 \mu \mathrm{mol} / \mathrm{L} \mathrm{A} \beta 40), 1: 0(0.1 \mu \mathrm{mol} / \mathrm{L} \mathrm{A} \beta 42$ alone) and 4:0 (0.4 $\mu \mathrm{mol} / \mathrm{L} \mathrm{A} \beta 42$ alone). Consistent with the previous finding, the increase in the $A \beta 42 / 40$ ratio from $1: 9$ to $4: 6$ resulted in the conformational change of PS1 toward the pathogenic state. Although a trend toward more severe change in PS1 conformation was observed in the 4:0-treated cells (A $\beta 42: A \beta 40)$, compared with that with the $4: 6$ treatment $(A \beta 42: A \beta 40)$, no statistically significant difference between neurons treated with the $A \beta 42$ to $A \beta 40$ ratios at $4: 6$ versus 1:0 versus 4:0 was observed (all had "closed" PS1 conformation). Intriguingly, even though the absolute amount of $A \beta 42$ was the same in the $\mathrm{A} \beta 42: \mathrm{A} \beta 40$ at the $1: 9$ and 1:0 conditions (0.1 $\mu \mathrm{mol} / \mathrm{L})$, PS1 adapted closed, pathogenic conformation only in the latter (Figure 2B). These data would support the hypothesis that the ratio of the $\mathrm{A} \beta 42 / 40$ rather than the absolute $\mathrm{A} \beta 42$ level is crucial for PS1 conformational change and imply that presence of the $A \beta 40$ might decrease the pathogenic effect of the longer A $\beta$ species on PS1 conformation.

\section{Increased Intracellular Calcium Load Due to High A $342 / 40$ Ratio Leads to Pathogenic Shift in PS1 Conformation}

Next we investigated the potential mechanisms by which $A \beta 42$ and $A \beta 40$ peptides at the high $A \beta 42 / 40$ ratio might cause the pathogenic change within the
PS1 subdomain architecture. First, to exclude the possibility that the observed effect is due to neurotoxicity of the applied $A \beta$ treatment, we monitored the level of lactate dehydrogenase in conditioned medium from the control and A $\beta$-treated neurons. We determined that treatments with the given $\mathrm{A} \beta$ concentrations, irrespective of the $\mathrm{A} \beta 42 / 40$ ratio, did not cause any significant cytotoxicity (Figure 3).

As $A \beta$ has been linked to impaired $\mathrm{Ca}^{2+}$ homeostasis, we assayed calcium levels in neurons treated with the 1:9, $3: 7,4: 6$ or $10: 0$ ratios of $A \beta 42: A \beta 40$ using an Indo-1 reporter probe. Elevated intracellular calcium levels were observed only after the treatment with $A \beta$ mixtures containing $A \beta 42$ and $A \beta 40$ peptides in the $4: 6$ and 10:0 ratios (Figure $4 \mathrm{~A}$ ). The increase in the intracellular calcium load correlated with the higher FRET efficiency between fluorescently labeled PS1 domains (Figure 4B).

To confirm that the observed effects are physiologically relevant, we conducted additional experiments where $\mathrm{A} \beta$ concentration was reduced to the nanomolar range. For these we chose to compare the effect of the mixtures containing $A \beta 42$ and $A \beta 40$ at the 1:9 and 4:6 ratios, that showed distinct $A \beta$-mediated effects on intracellular PS1 conformation (Figure 2) and intracellular $\mathrm{Ca}^{2+}$ (Figure 4A). The neurons incubated with the medium containing $A \beta 42$ and $\mathrm{A} \beta 40$ at the $4 \mathrm{nmol} / \mathrm{L}$ and $6 \mathrm{nmol} / \mathrm{L}$ concentrations, respectively, (4:6 ratio) presented higher intracellular calcium levels and altered conformation of PS1 when compared with the neurons treated with the mixture of $1 \mathrm{nmol} / \mathrm{L} \mathrm{A} \beta 42$ and $9 \mathrm{nmol} / \mathrm{L}$ A $\beta 40$ (1:9 ratio) (Supplementary Figure S2). This supports the physiological relevance of the observed A $\beta$-mediated effects.

To verify the effect of calcium influx on the conformational shift of endogenous PS1, we stimulated primary neurons with $50 \mathrm{mmol} / \mathrm{L} \mathrm{KCl}$ and assayed the proximity between fluorophores labeling the PS1 N-terminus and the PS1 loop domain using antibody-based 


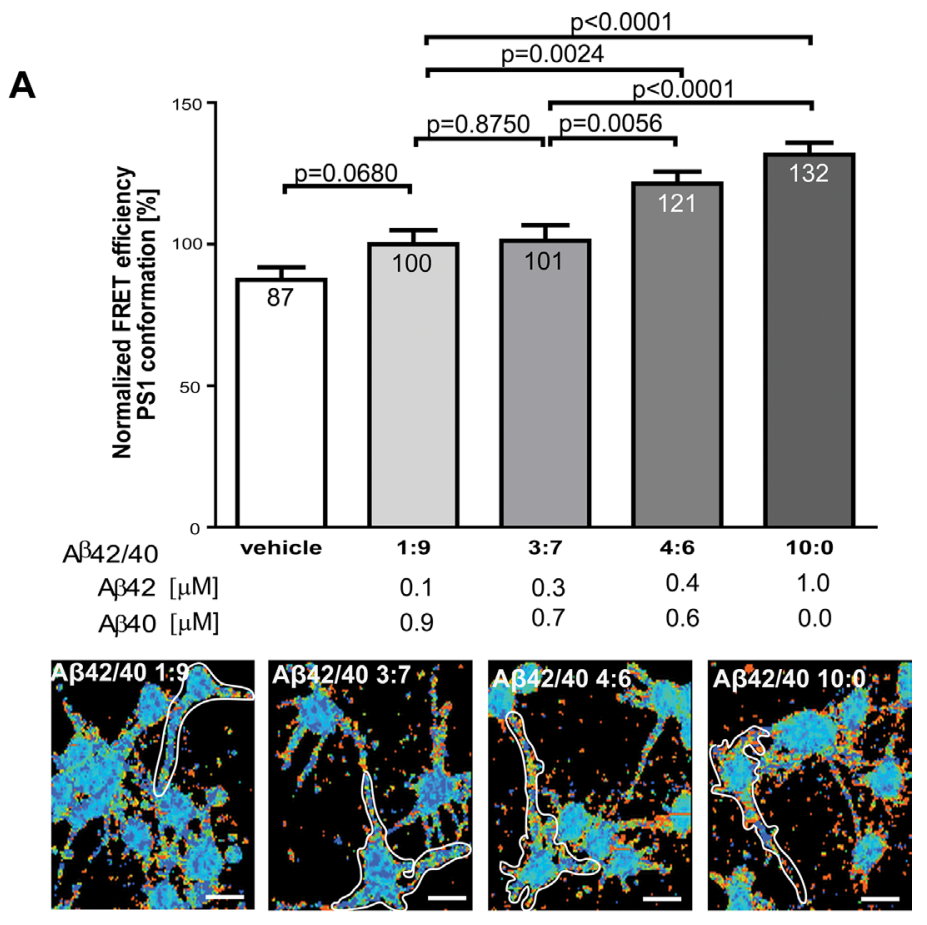

B

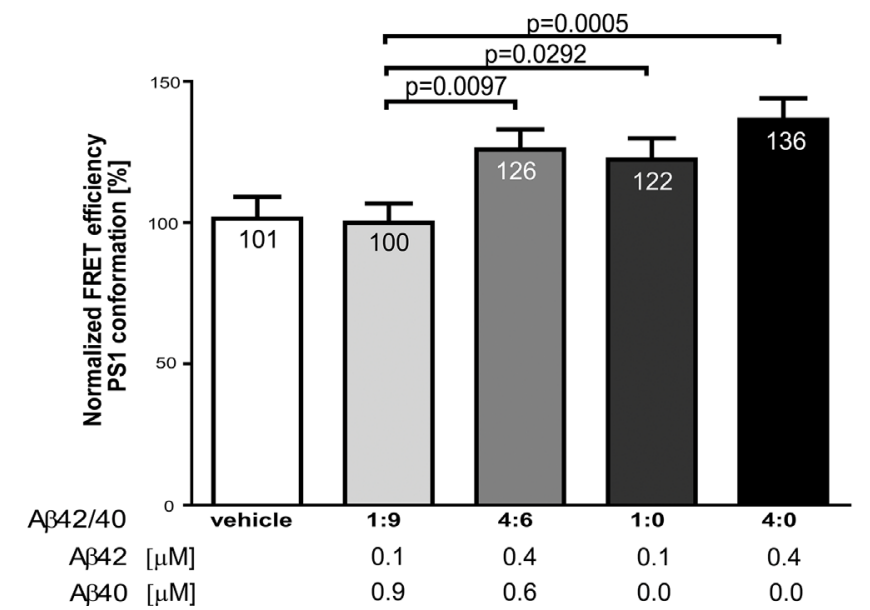

Figure 2. Effect of $A \beta 42 / 40$ ratio on PS1 conformation. (A) FLIM analysis of the PS1 conformation in primary neurons pretreated for $2 \mathrm{~h}$ with the mixtures of $A \beta 42$ and $A \beta 40$ peptides at $(A)$ the varied ratios at the $1 \mu \mathrm{mol} / L$ total $A \beta$ concentration or $(B)$ the varied ratios ( $1: 9$ or $4: 6$ ) at the $1 \mu \mathrm{mol} / \mathrm{L}$ total $A \beta$ concentration or different absolute amounts of $A \beta 42$ $(0.1 \mu \mathrm{mol} / \mathrm{L}$ or $0.4 \mu \mathrm{mol} / \mathrm{L})$. The graphs present the increase in FRET efficiency, corresponding to "closed" PS1 conformation, in neurons treated with $A \beta 42$ to $A \beta 40$ at the relatively high ratios. (A) $n=73$ (vehicle), $n=77$ (1:9), $n=74$ (3:7), $n=157$ (4:6), $n=152$ (10:0), (B) $n=50$ (vehicle), $n=50$ (1:9), $n=49(4: 6), n=51(1: 0), n=50(4: 0), n=$ total number of neurons analyzed from three independent experiments, mean $\pm S E M$; two-tailed unpaired Student $t$ test. The data are normalized to the values recorded in neurons pretreated with $A \beta 42$ and $A \beta 40$ peptides at the 1 to 9 ratio. The images present color-coded lifetime of the donor fluorophore. The red pixels correspond to the shorter donor fluorophore lifetimes, indicative of higher FRET efficiency (relative proximity) between the fluorescently labeled PS1 domains. The scale bar corresponds to $10 \mu \mathrm{m}$. A $\beta$ - amyloid $\beta$; PS1 - presenilin 1; FRET - Förster resonance energy transfer.
FLIM. An increase in the intracellular calcium levels and the shift of PS1 toward the pathogenic state were recorded in $\mathrm{KCl}$-treated neurons (Figures 5A, B). Importantly our previous data demonstrating an increase in the $\mathrm{A} \beta 42 / 40$ ratio upon the rise in intracellular calcium (20) support the functional relevance of the observed shift within the PS1 subdomain organization.

To further determine if the increase in the intracellular calcium load is indeed causative of the conformational shift of PS1, we applied another calcium elevating agent, A2317 calcium ionophore, to the primary neurons overexpressing G-PS1-R probe as a reporter of the PS1 conformation. Application of the A2317 resulted in a marked rise in the intracellular calcium load (Figure 5C), which corresponded to the increased FRET efficiency between the GFP and RFP labeling the respective PS1 domains, compared with the control (Figure 5D). This argues in favor of the relationship between the elevated calcium and pathogenic conformational changes within the PS1 molecule.

\section{DISCUSSION}

As PS1 $\gamma$-secretase is the enzyme responsible for the final cut of the amyloid precursor protein (APP) substrate producing A $\beta$ peptides, altered PS1 structure and function would affect the composition of $A \beta$ species generated. The $A \beta$ aggregates in the brain are surrounded by significantly altered microenvironment exhibiting gliosis and an increased level of reactive oxygen species and calcium dyshomeostasis and a substantial accumulation of the soluble oligomeric $A \beta$ in a halo enclosing the plaque core (22-25). However, the crosstalk between all these insults and PS1 architecture remains poorly understood.

The current study demonstrates that there is a reciprocal relationship between elevated $A \beta 42$ or $A \beta 42 / 40$ ratio and the pathogenic conformational state of PS1. Our data imply that an increased $A \beta 42 / 40$ ratio could trigger a conformational shift of PS1 toward the 


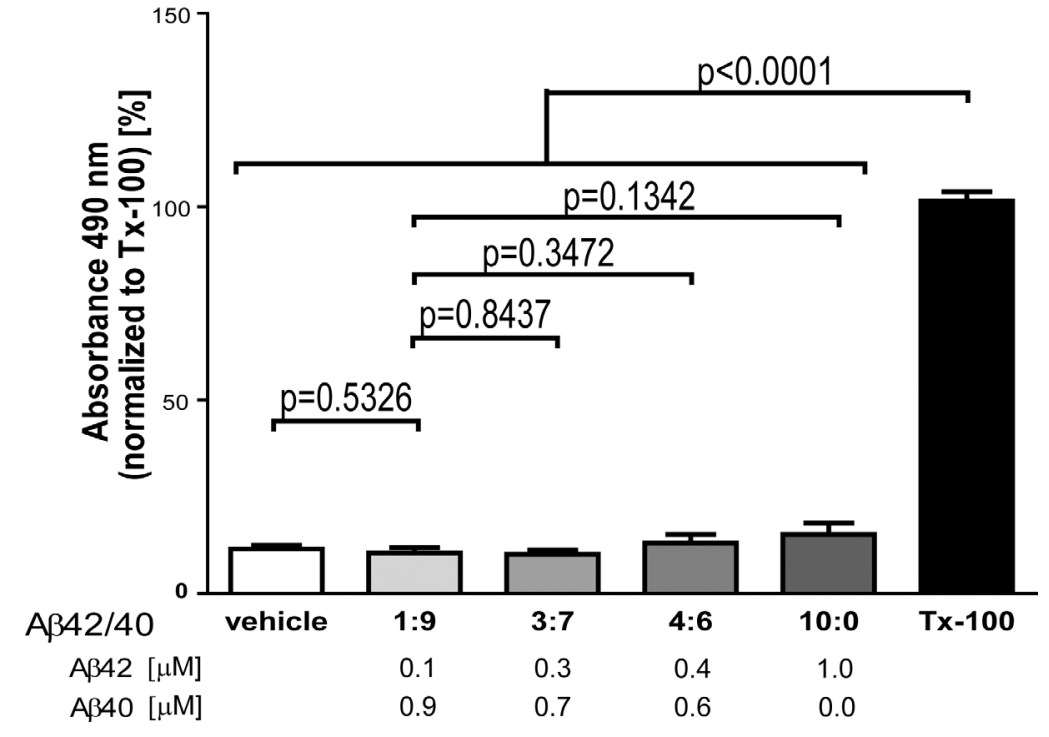

Figure 3. Cytotoxicity of A $\beta$ treatment. Relative levels of the lactate dehydrogenase (LDH) activity in the conditioned medium after $A \beta$ treatments. $1 \% \mathrm{Tx}-100$ is used as a positive control. $n=21$ (vehicle), $n=18$ (1:9), $n=15$ (3:7), $n=18(4: 6), n=17$ (10:0), $n=18$ (Tx-100), mean \pm SEM, two-tailed unpaired Student $t$ test. $A \beta$ - amyloid $\beta$, Tx-100 - Triton X-100.

pathogenic state by means of increasing intracellular calcium load. This is in line with the data suggesting the importance of the A $\beta 42 / 40$ ratio in the AD progression and proposing calcium dyshomeostasis as one of the culprits exacerbating AD pathology (26-28).

Impairments in intracellular calcium can serve as an insult, provoking several alterations in the neuronal functions including activation of mitochondrial stress responses (29), disruption of spinodendritic signaling interface (30) and apoptosis (31), and can also accelerate A $\beta$ fibril formation (32). The latter would suggest an existence of a feed-forward mechanism of calcium impairments and toxic $A \beta$ generation. Our findings point toward yet another possible mechanism spinning the vicious cycle. We propose that $A \beta$ peptides at the elevated ratio of $A \beta 42$ to $A \beta 40$, which results from the pathogenically altered cleavage of the amyloid precursor protein (APP) by PS1 (8), increase the intracellular calcium load, which could in turn modulate conformation of the PS1 enzyme.

Several mechanisms can be implicated in the calcium-mediated pathogenic reorganization of PS1. One possibility is that calcium influx results in the rearrangement of the PS1-interacting partners, which triggers different degrees of "squishing" of the highly flexible PS1/ $\gamma$-secretase. Alternatively, the calcium influx may induce posttranslational modifications in PS1. Such posttranslational modifications may result in the rearchitecture by altering local charges and atomic forces within the PS1 molecule.

An atomic structure of the $\gamma$-secretase complex at $3.4 \AA$ resolution has recently been reported (33). However, the precise molecular reshuffles of the PS1 domains on the atomic level caused by various factors remain unknown. Recent studies using cysteine cross-linking have demonstrated a correlation between the distance of the cytosolic residues in PS1 transmembrane domains 4 and 7 and $\mathrm{A} \beta 42$ generation, proposing that the $\mathrm{dy}$ namics of this region might be impaired by PS1 mutations (34). In addition, simulated modeling of the dynamic repositioning of the PS1 cytosolic loop suggested that it may control substrate access and cleavage precision, and these arrangements within the PS1 subdomain can be affected by fAD mutations (35). The PS1 conformation assay that we employ in this study detects relative changes between the cytosolic loop and PS1 N-terminus in response to $A \beta$ in intact neurons. Although the FRET-based PS1 conformation assay applied here does not address the detailed structural rearrangements within the PS1 molecule, it can reliably distinguish between the "normal" and "pathogenic" PS1 conformations in situ, as determined by us and others $(11,13,17,36)$. The relatively small changes observed in the FRET efficiency, reflecting proximity between fluorescent tags on PS1, may translate into much greater alterations in PS1 on the sub-molecular scale. It is highly likely that even small changes that persist over a long time may have a detrimental effect.

Our findings also indicate that $\mathrm{A} \beta 40$ may decrease the pathogenic effect of A $\beta 42$ on PS1 conformation, suggesting that it could serve as a protective factor under a certain threshold of $\mathrm{A} \beta 42$. We found that adding $0.9 \mu \mathrm{mol} / \mathrm{L} \mathrm{A} \beta 40$ to the media containing $0.1 \mu \mathrm{mol} / \mathrm{L} \mathrm{A} \beta 42$ prevented the $A \beta 42$-induced pathogenic PS1 conformational change, even though the total $\mathrm{A} \beta$ concentration in this case was $\sim 10$ times higher than in $0.1 \mu \mathrm{mol} / \mathrm{L} \mathrm{A} \beta 42$ alone (Figure 2B). The potential protective effect of $A \beta 40$, however, has limits, and $0.6 \mu \mathrm{mol} / \mathrm{L}$ A $\beta 40$ no longer averts the detrimental effect of $0.4 \mu \mathrm{mol} / \mathrm{L}$ A $\beta 42$ on PS1 conformation (4:6 versus 4:0 A $\beta 42 / 40$ ratio). These data are in agreement with the previous reports suggesting a protective role of the $A \beta 40$ by showing that A $\beta 40$ could rescue A $\beta 42$-induced cell death in vitro $(10,37)$. The prevention of $A \beta 42$ aggregation and toxicity was proposed as a possible mechanism of the A $\beta 40$ effect. Together, these data support the idea that a reduced level of $A \beta 40$ and/or an increased $A \beta 42 / 40$ ratio in the brain, rather than the absolute $A \beta$ amount, may be detrimental factors in AD.

Importantly, our current findings suggest that $A \beta$ may trigger PS1 
A

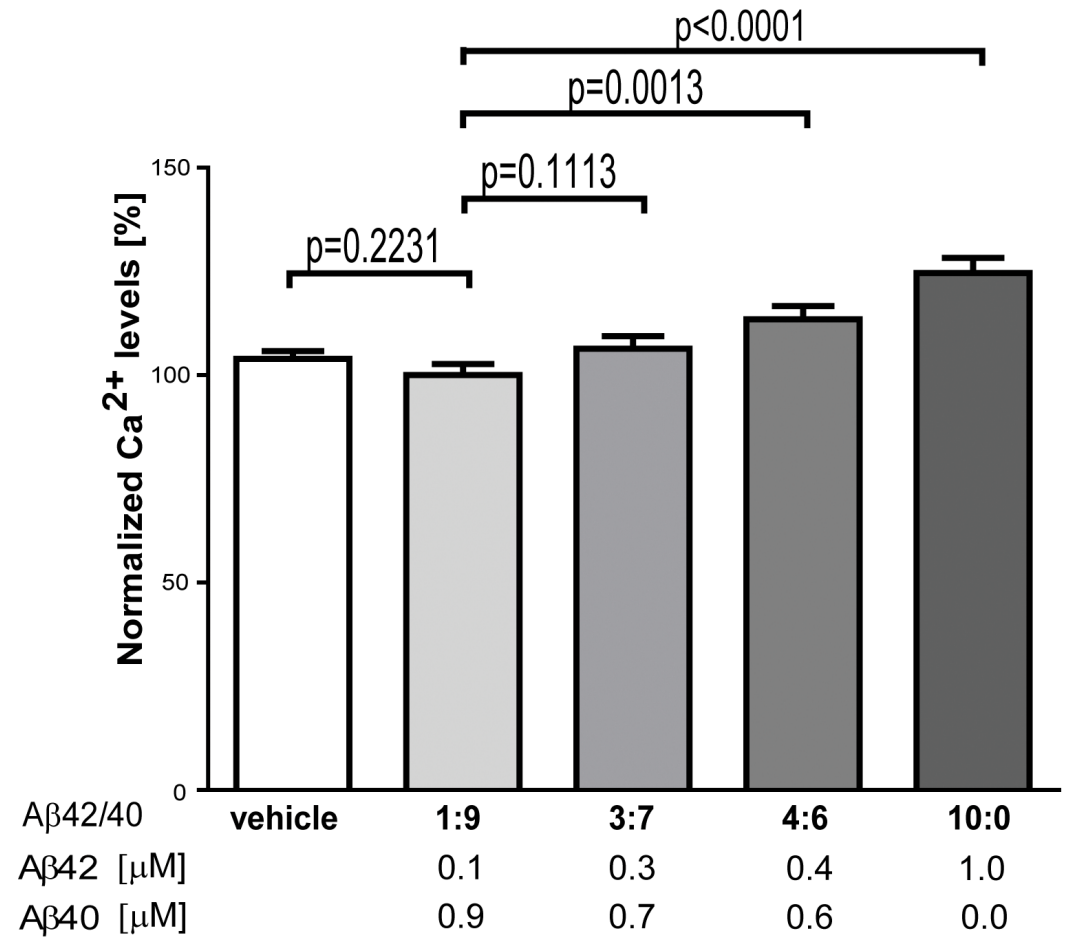

B

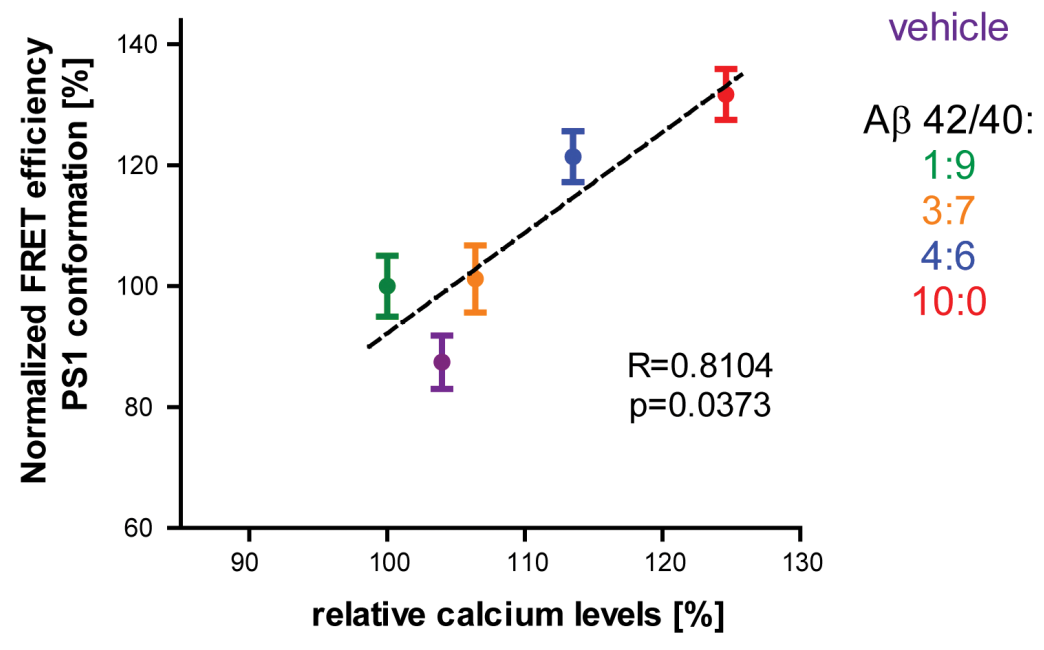

Figure 4. Intracellular calcium level upon A $\beta$ treatment. $(A)$ The relative levels of intracellular calcium in primary neurons were determined using ratiometric Indo-1 dye. The graph presents the increase in the calcium level upon treatment with $A \beta$ peptides at increasing A $342 / 40$ ratio. $n=234$ (vehicle), $n=243$ (1:9), $n=271$ (3:7), $n=263$ (4:6), $n=219$ (10:0), $n$ $=$ total number of neurons analyzed from three independent experiments, mean \pm SEM, two-tailed unpaired Student $t$ test. (B) Correlation between PS1 conformational changes (FRET efficiency) and intracellular calcium levels in primary neurons treated with vehicle or $A \beta 42$ to $A \beta 40$ at $1: 9 ; 3: 7 ; 4: 6$ or 10:0 ratios. Pearson's $X$-square test. $A \beta$ - amyloid $\beta ; P S 1$ - presenilin 1; FRET - Förster resonance energy transfer. conformational change associated with increased $A \beta 42$ production in the neighboring neurons, which would exacerbate and possibly incite propagation of the amyloid pathology. Although the observed alterations triggered by the relatively short $(2 \mathrm{~h})$ $\mathrm{A} \beta$ pretreatment in the reported experimental settings are comparatively small, the prolonged exposure to the pathogenic perturbations would result in the progressive development of the pathology.

In summary, our findings link three key players in $\mathrm{AD}$ pathogenesis, toxic A $\beta 42$ specie, PS1 $/ \gamma$-secretase and impairments in calcium homeostasis, provide novel insight into the progressive exacerbation of $\mathrm{AD}$ pathology, and point toward molecular events which might be targeted for therapeutic intervention.

\section{CONCLUSION}

Our findings demonstrate that extracellular amyloid $\beta(\mathrm{A} \beta)$ peptides, when present at a relatively high ratio of the longer to shorter (A $\beta 42$ to $A \beta 40)$ species, trigger an increase in the intracellular calcium level in the affected neuron. This leads to a pathogenic conformational shift in the endogenous PS $1 / \gamma$-secretase within the cell, favoring further production of the longer $A \beta$ peptides, and results in an increased ratio of $A \beta 42$ to A $\beta 40$. These events can occur reciprocally in $A \beta$-producing cells as well as in the neighboring neurons, demonstrating a noncell autonomous mechanism of $\mathrm{A} \beta$ action. The discovery of the novel mechanism of potential exacerbation of the amyloid pathology may facilitate the design of new therapeutic approaches focused on inhibition of the disease progression by "stabilizing" PS1 conformation.

\section{ACKNOWLEDGMENTS}

We thank Shuko Takeda for guidance with preparation of the $A \beta$ peptides and Sarah Svirsky for help with primary neuronal cultures. The work was supported by NIH grants AG 044486 and AG 15379 (OB). 
A
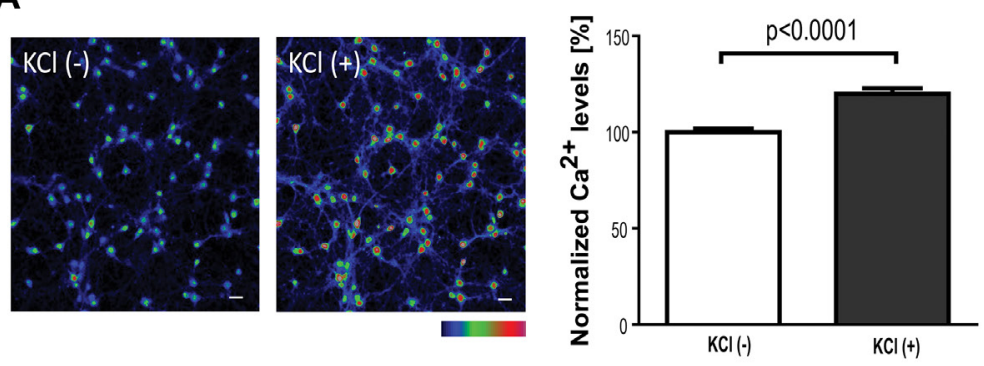

B

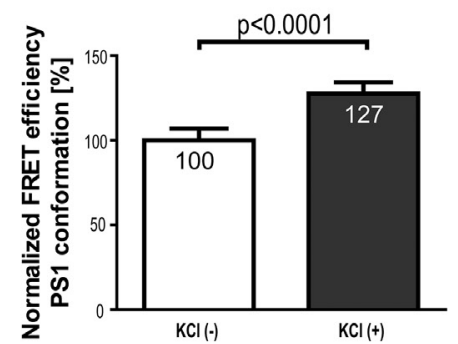

C
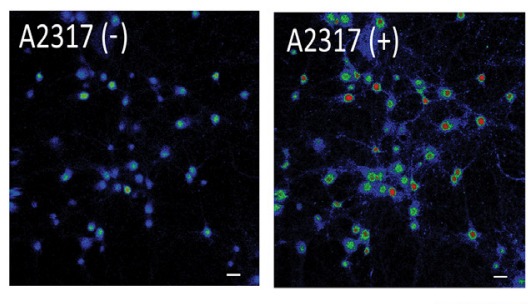

D

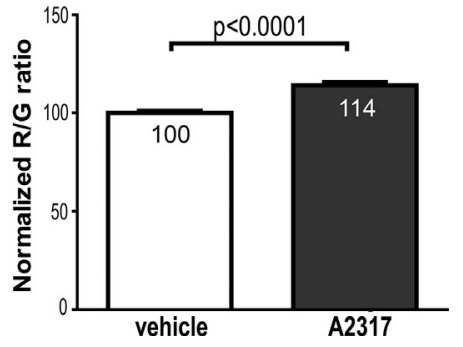

Figure 5. $\mathrm{Ca}^{2+}$ overload triggers conformational change of PS1. (A) Increase in the intracellular calcium levels in neurons treated with $50 \mathrm{mmol} / \mathrm{L} \mathrm{KCl}$ is monitored by Oregon Green BAPTA-1/AM (images) or by Indo-1 ratiometric probe (graph). The pseudo-colored images show intracellular calcium levels pre- and post-treatment. The red pixels correspond to higher calcium levels. The graph presents quantification of the intracellular calcium levels. $n=310(\mathrm{KCl}-) n=251(\mathrm{KCl}+), n=$ total number of neurons analyzed from three independent experiments mean \pm SEM, two-tailed unpaired Student $t$ test. The scale bar corresponds to $20 \mu \mathrm{m}$. (B) FLIM analysis of the PS1 conformation pre- and post-KCl treatment in neurons. Increase in the FRET efficiency, reflective of pathogenic "closed" PS1 conformation, is detected after 15 min of $\mathrm{KCl}$ treatment. $\mathrm{n}=84(\mathrm{KCl}-) \mathrm{n}=85$ $(\mathrm{KCl}+), \mathrm{n}=$ total number of neurons analyzed from 3 independent experiments, mean $\pm \mathrm{SEM}$, two-tailed unpaired Student $t$ test. The data are normalized to the values recorded in the neurons in $\mathrm{KCl}$ - conditions. (C) Increase in the intracellular calcium levels in neurons treated with $5 \mu \mathrm{mol} / \mathrm{L}$ A2317 calcium ionophore monitored by Oregon Green BAPTA-1/AM. The pseudo-colored images show intracellular calcium levels pre- and post-treatment. The red pixels correspond to higher calcium levels. The scale bar corresponds to $20 \mu \mathrm{m}$. (D) Spectral FRET analysis of the PS1 conformation pre- and post-A2317 calcium ionophore treatment in primary neurons. Increase in FRET efficiency ( $R / G$ ratio) reflective of pathogenic "closed" PS1 conformation is detected in A2317-treated neurons. $n=180$ (vehicle) $n=185$ (A2317), $n=$ total number of neurons analyzed from three independent experiments, mean \pm SEM, two-tailed unpaired Student $t$ test. A $\beta$ - amyloid $\beta ; P S 1-$ presenilin 1; FRET - Förster resonance energy transfer.

\section{DISCLOSURE}

The authors declare they have no competing interests as defined by Molecular Medicine, or other interests that might be perceived to influence the results and discussion reported in this paper.

\section{REFERENCES}

1. Hardy J, Selkoe DJ. (2002) The amyloid hypothesis of Alzheimer's disease: progress and problems on the road to therapeutics. Science. 297:353-6.

2. Braak H, Braak E, Ohm T, Bohl J. (1989) Alzheimer's disease: mismatch between amyloid plaques and neuritic plaques. Neurosci. Lett. 103:24-28.

3. Takami M, et al. (2009) gamma-Secretase: successive tripeptide and tetrapeptide release from the transmembrane domain of beta-carboxyl terminal fragment. J. Neurosci. 29:13042-52.

4. Roychaudhuri R, et al. (2013) C-terminal turn stability determines assembly differences between Abeta40 and Abeta42. J. Mol. Biol. 425:292-308.

5. Ran $Y$, et al. (2014) gamma-Secretase processing and effects of gamma-secretase inhibitors and modulators on long Abeta peptides in cells. J. Biol. Chem. 289:3276-87.

6. Chen YR, Glabe CG. (2006) Distinct early folding and aggregation properties of Alzheimer amyloid-beta peptides Abeta40 and Abeta42: stable trimer or tetramer formation by Abeta42. J. Biol. Chem. 281:24414-22.

7. Klein AM, Kowall NW, Ferrante RJ. (1999) Neurotoxicity and oxidative damage of beta amyloid 1-42 versus beta amyloid 1-40 in the mouse cerebral cortex. Ann. N. Y. Acad. Sci. 893:314-20.

8. Fernandez MA, Klutkowski JA, Freret T, Wolfe MS. (2014) Alzheimer presenilin-1 mutations dramatically reduce trimming of long amyloid beta-peptides (Abeta) by gamma-secretase to increase 42-to-40-residue Abeta. J. Biol. Chem. 289:31043-52.

9. Kuperstein I, et al. (2010) Neurotoxicity of Alzheimer's disease Abeta peptides is induced by small changes in the Abeta42 to Abeta40 ratio. EMBO J. 29:3408-20.

10. Jan A, Gokce O, Luthi-Carter R, Lashuel HA. (2008) The ratio of monomeric to aggregated forms of Abeta40 and Abeta42 is an important determinant of amyloid-beta aggregation, fibrillogenesis, and toxicity. J. Biol. Chem. 283:28176-89.

11. Uemura K, et al. (2009) Allosteric modulation of PS1/gamma-secretase conformation correlates with amyloid beta(42/40) ratio. PloS One. 4:e7893.

12. Elad N, et al. (2015) The dynamic conformational landscape of gamma-secretase Nadav. J. Cell Science. 128:589-98.

13. Berezovska O, et al. (2005) Familial Alzheimer's disease presenilin 1 mutations cause alterations in the conformation of presenilin and interactions with amyloid precursor protein. J. Neurosci. 25:3009-17. 
14. Ingelsson M, et al. (2004) Early Abeta accumulation and progressive synaptic loss, gliosis, and tangle formation in AD brain. Neurology. 62:925-31.

15. Wahlster L, et al. (2013) Presenilin-1 adopts pathogenic conformation in normal aging and in sporadic Alzheimer's disease. Acta Neuropathol. 125:187-99.

16. Placanica L, Zhu L, Li YM. (2009) Gender- and age-dependent gamma-secretase activity in mouse brain and its implication in sporadic Alzheimer disease. PloS One 4:e5088.

17. Lleo A, et al. (2004) Nonsteroidal anti-inflammatory drugs lower Abeta42 and change presenilin 1 conformation. Nat. Med. 10:1065-6.

18. Ohki Y, et al. (2011) Phenylpiperidine-type gamma-secretase modulators target the transmembrane domain 1 of presenilin 1. EMBO J. 30:4815-24.

19. Takeda S, et al. (2013) Brain interstitial oligomeric amyloid beta increases with age and is resistant to clearance from brain in a mouse model of Alzheimer's disease. FASEB J. 27:3239-48.

20. Kuzuya A, et al. (2016) Identification of the novel activity-driven interaction between synaptotagmin 1 and presenilin 1 links calcium, synapse, and amyloid beta. BMC Biol. 14:25.

21. Wu HY, et al. (2012) Distinct dendritic spine and nuclear phases of calcineurin activation after exposure to amyloid-beta revealed by a novel fluorescence resonance energy transfer assay. J. Neurosci. 32:5298-309.

22. Kuchibhotla KV, Lattarulo CR, Hyman BT, Bacskai BJ. (2009) Synchronous hyperactivity and intercellular calcium waves in astrocytes in Alzheimer mice. Science. 323:1211-5.

23. Xie H, et al. (2013) Mitochondrial alterations near amyloid plaques in an Alzheimer's disease mouse model. J. Neurosci. 33:17042-51.

24. Koffie RM, et al. (2009) Oligomeric amyloid beta associates with postsynaptic densities and correlates with excitatory synapse loss near senile plaques. Proc. Natl. Acad. Sci. U. S. A. 106:4012-7.

25. Osborn LM, Kamphuis W, Wadman WJ, Hol EM. (2016) Astrogliosis: An integral player in the pathogenesis of Alzheimer's disease. Prog. Neurobiol.. [Epub ahead of print].

26. Green KN. (2009) Calcium in the initiation, progression and as an effector of Alzheimer's disease pathology. J. Cell. Mol. Med. 13:2787-99.

27. Demuro A, Parker I, Stutzmann GE. (2010) Calcium signaling and amyloid toxicity in Alzheimer disease. J. Biol. Chem. 285:12463-8.

28. Bezprozvanny I. (2009) Calcium signaling and neurodegenerative diseases. Trends Mol. Med. 15:89-100.

29. Votyakova TV, Reynolds IJ. (2005) Ca2+-induced permeabilization promotes free radical release from rat brain mitochondria with partially inhibited complex I. J. Neurochem. 93:526-37.

30. Kuchibhotla KV, et al. (2008) Abeta plaques lead to aberrant regulation of calcium homeostasis in vivo resulting in structural and functional disruption of neuronal networks. Neuron. 59:214-25.
31. Takei N, Endo Y. (1994) Ca2+ ionophore-induced apoptosis on cultured embryonic rat cortical neurons. Brain Res. 652:65-70.

32. Isaacs AM, Senn DB, Yuan M, Shine JP, Yankner BA. (2006) Acceleration of amyloid beta-peptide aggregation by physiological concentrations of calcium. J. Biol. Chem. 281:27916-23.

33. Bai XC, et al. (2015) An atomic structure of human gamma-secretase. Nature. 525:212-7.

34. Tominaga A, Cai T, Takagi-Niidome S, Iwatsubo T, Tomita T. (2016) Conformational changes in transmembrane domain 4 of presenilin 1 are associated with altered amyloid-beta 42 production. J. Neurosci. 36:1362-72.

35. Somavarapu AK, Kepp KP. (2016) The dynamic mechanism of presenilin-1 function: Sensitive gate dynamics and loop unplugging control protein access. Neurobiol. Dis. 89:147-56.

36. Wang X, et al. (2015) gamma-Secretase Modulators and Inhibitors Induce Different Conformational Changes of Presenilin 1 Revealed by FLIM and FRET. J. Alzheimers Dis. 47:927-37.

37. Zou K, et al. (2003) Amyloid beta-protein (Abeta)1-40 protects neurons from damage induced by Abeta1-42 in culture and in rat brain. J. Neurochem. 87:609-19.

Cite this article as: Zoltowska KM, Maesako M, Berezovska O. (2016) Interrelationship between changes in the amyloid $\beta 42 / 40$ ratio and presenilin 1 conformation. Mol. Med. 22:329-37. 